\title{
Multi-site evaluation of a computer aided detection (CAD) algorithm for small Acute Intra-cranial Hemorrhage and development of a stand-alone CAD system ready for deployment in a clinical environment
}

\author{
Ruchi R. Deshpande ${ }^{a}$, James Fernandez ${ }^{a}$, Joon K. Lee ${ }^{a}$, Tao Chan ${ }^{b}$, Brent J. Liu ${ }^{a}$, H.K. Huang ${ }^{a}$ \\ amage Processing and Informatics Laboratory, Department of Radiology, \\ 2250 Alcazar Street, CSC 105, Los Angeles, CA 90033 \\ ${ }^{\mathrm{b}}$ Dept. of Health Technology and Informatics, The Hong Kong Polytechnic University, Hong \\ Kong, SAR, China
}

\begin{abstract}
Timely detection of Acute Intra-cranial Hemorrhage (AIH) in an emergency environment is essential for the triage of patients suffering from Traumatic Brain Injury. Moreover, the small size of lesions and lack of experience on the reader's part could lead to difficulties in the detection of AIH. A CT based CAD algorithm for the detection of AIH has been developed in order to improve upon the current standard of identification and treatment of AIH. A retrospective analysis of the algorithm has already been carried out with 135 AIH CT studies with 135 matched normal head CT studies from the Los Angeles County General Hospital/ University of Southern California Hospital System (LAC/USC). In the next step, AIH studies have been collected from Walter Reed Army Medical Center, and are currently being processed using the AIH CAD system as part of implementing a multi-site assessment and evaluation of the performance of the algorithm. The sensitivity and specificity numbers from the Walter Reed study will be compared with the numbers from the LAC/USC study to determine if there are differences in the presentation and detection due to the difference in the nature of trauma between the two sites.. Simultaneously, a stand-alone system with a user friendly GUI has been developed to facilitate implementation in a clinical setting.
\end{abstract}

Keywords: Computer Aided Detection, Acute Inter-cranial Hemorrhage, CT, Brain

\section{INTRODUCTION}

Acute Intra-cranial hemorrhage (AIH) can lead to significant morbidity and mortality unless detected and treated in a timely manner. Heavy emphasis is placed on AIH detection and identification in patients suffering from head trauma and neurological disturbances since it has a direct bearing on further management and treatment strategies. This is especially so in the military and in emergency rooms where triage of patients is crucial. This vital step is often carried out initially by emergency physicians, internists and neurosurgeons. It has been shown that the competence provided by these acute care physicians in reading brain CT scans may not be optimum [1]. Since CT is the modality of choice in diagnosing AIH $[2,3]$, the challenge can be remedied by a computer aided detection system which targets these physicians and enhances their brain CT interpretations. In the event of the AIH lesions being too small or inconspicuous, this system augments the skills of both acute care physicians and radiologists in addition to rendering consistency to their interpretations.

\section{THE CAD ALGORITHM}

The CAD algorithm for AIH detection was developed and tested with MATLAB (The MathWorks, Inc., Natick, MA, USA). The algorithm accepts a series of CT images in the DICOM format, as input. The system output is again a series of DICOM images, which are secondary captures of the Computer Aided Detection. These images graphically highlight the areas positively identified by the algorithm as AIH lesions. Figure 1 depicts a flowchart of the algorithm, and Table 1 summarizes the image processing techniques involved in each step.

Medical Imaging 2010: Advanced PACS-based Imaging Informatics and Therapeutic Applications, edited by Brent J. Liu, William W. Boonn, Proc. of SPIE Vol. 7628, 76280H · (c) 2010 SPIE

CCC code: $1605-7422 / 10 / \$ 18 \cdot$ doi: $10.1117 / 12.844833$ 


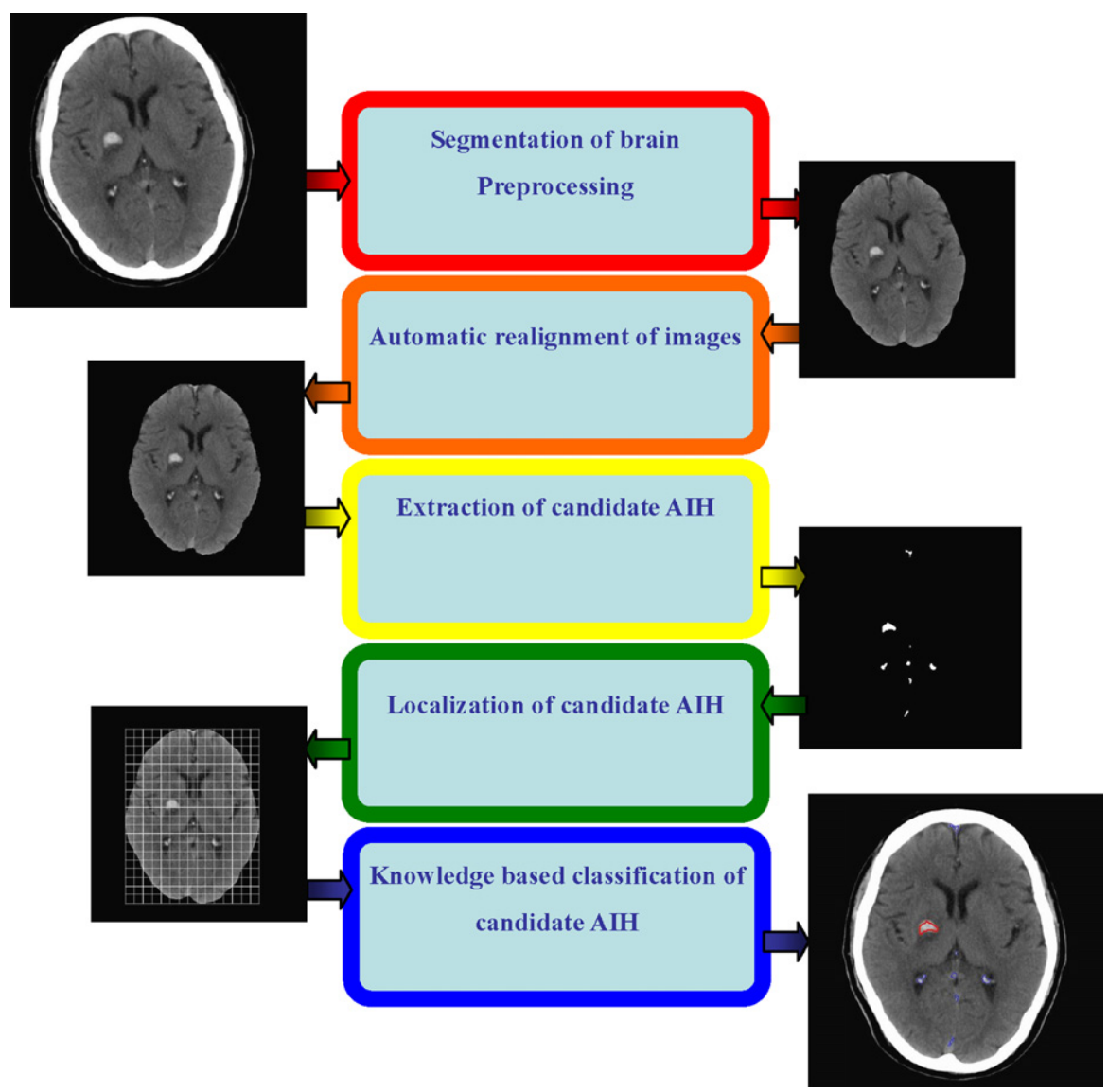

Figure 1: Schematic diagram of the CAD system. Intermediary outputs of an image showing right basal ganglia hemorrhage illustrate the effect of individual steps [4].

Table 1: Details of individual image processing and analysis steps in the CAD system outlined in Figure 1 [4]

\begin{tabular}{|c|c|c|}
\hline Steps & Methods & Purposes \\
\hline $\begin{array}{l}\text { Segmentation of } \\
\text { intracranial contents }\end{array}$ & $\begin{array}{l}\text { Global thresholding and morphological operations } \\
\text { Remove structures not contiguous with the main } \\
\text { central bulk of intracranial contents }\end{array}$ & $\begin{array}{l}\text { Remove bones of skull and face } \\
\text { Remove scalp, orbits, and other head and } \\
\text { neck soft tissue }\end{array}$ \\
\hline $\begin{array}{l}\text { Preprocessing of } \\
\text { intracranial contents }\end{array}$ & $\begin{array}{l}\text { Median filtering } \\
\text { Adjustment of intensity according to distance from the } \\
\text { skull }\end{array}$ & $\begin{array}{l}\text { Denoising } \\
\text { Correction for CT cupping artifacts }\end{array}$ \\
\hline $\begin{array}{l}\text { Automatic } \\
\text { realignment of } \\
\text { images }\end{array}$ & $\begin{array}{l}\text { Automatic localization of limits of brain, ventricles, } \\
\text { floor of anterior intracranial fossa, mid-sagittal plane }\end{array}$ & Align the brain into normal position \\
\hline $\begin{array}{l}\text { Extraction of } \\
\text { candidate AIH }\end{array}$ & $\begin{array}{l}\text { Top-hat transformation } \\
\text { Subtraction between the two sides }\end{array}$ & $\begin{array}{l}\text { Highlight local high density regions } \\
\text { Extract asymmetrically high density regions }\end{array}$ \\
\hline $\begin{array}{l}\text { Localization of } \\
\text { candidate AIH }\end{array}$ & $\begin{array}{l}\text { Registration of the brain in question against a } \\
\text { normalized coordinate system }\end{array}$ & $\begin{array}{l}\text { Render the candidate AIH anatomical } \\
\text { information }\end{array}$ \\
\hline $\begin{array}{l}\text { Knowledge-based } \\
\text { classification of AIH }\end{array}$ & $\begin{array}{l}\text { Rule-based system with inputs of image features and } \\
\text { anatomical coordinates of the extracted candidates }\end{array}$ & $\begin{array}{l}\text { Distinguish genuine AIH from false positives } \\
\text { resulting from noise, artifacts, and normal } \\
\text { variants }\end{array}$ \\
\hline
\end{tabular}




\section{DEVELOPMENT OF A CAD SYSTEM FOR INTEGRATION WITH A CLINICAL ENVIRONMENT}

The previous implementation of the CAD algorithm was a static MATLAB program which needed to be run manually for each series in each study individually; following which, the results were pulled up and linked to the unprocessed images by the user. There was no facility for cataloguing image metadata; no service which maintains a link between the unprocessed and processed studies; and no interface for viewing processed results. The goal of building an automated system is to facilitate integration of a stand-alone CAD system into a clinical environment for testing, and ultimately, for long term use.

\subsection{Design issues to be considered when building an automated system for CAD}

(1) Ensuring that only the appropriate studies sent by PACS to the workstation get accepted. For instance, this application should only accept CT head image studies.

(2) Once accepted, they must be sorted and stored in a suitable file folder hierarchy and relevant metadata must be recorded somewhere for future reference

(3) A continuous check must be carried out for recently acquired images in order to process studies as and when they arrive

(4) There needs to be a mechanism to post-process the results to resolve any metadata conflicts, and to store the results in a pre-determined manner such that the resultant series are linked to the unprocessed, original series.

(5) The CAD process needs to run continuously in the background. On the front end, a graphical user interface is required to enable browsing through the acquired and processed studies, and to attach notes or comments to the results.

(6) A web server is required for remote access to the CAD workstation.

\subsection{System Architecture}

The automated CAD package is comprised of the following components integrated into one system, the components of which will be described in more detail below, each component being represented by the Red Circle Numbering as shown in Figure 2.

\section{DICOM Receiver}

The DICOM Receiver is a service which listens on a specified port for incoming DICOM studies and routes them to a pre-specified folder - the "raw" repository. This is the root folder that the control service scans every 5 minutes to check for new images.

\section{Pre-processing unit}

The studies received from PACS may not be ready for being processed by the CAD algorithm. The studies may be non brain CT studies, sent to the CAD workstation erroneously. Such studies need to be weeded out before they crash the system. The DICOM metadata of the received images must be scanned to ensure that it will be accepted by the algorithm. Once these conditions are satisfied, the images may be passed on to the Control Service. 


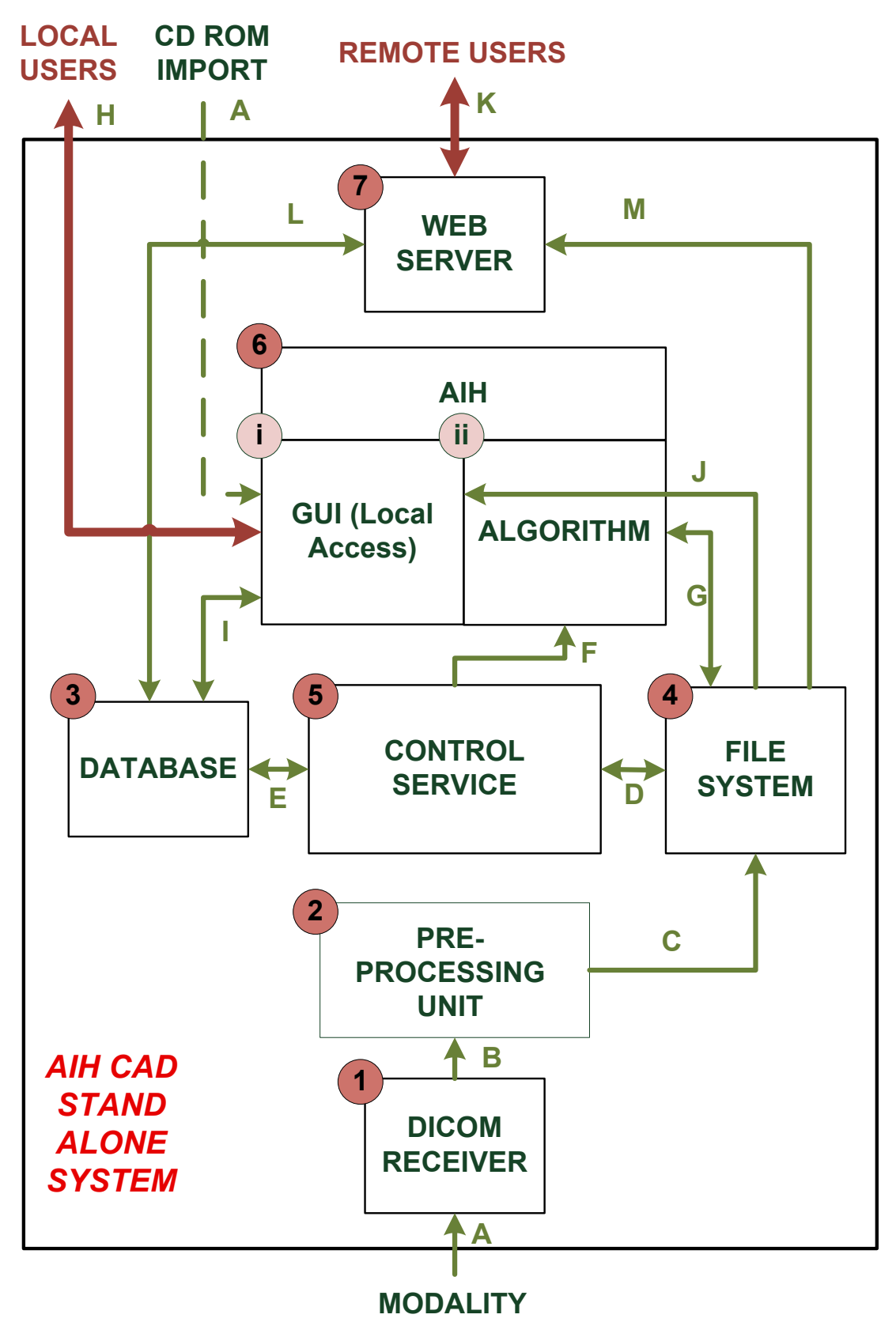

Figure 2: Architecture and workflow diagram for an automated Acute Inter-cranial Hemorrhage Computer Aided Diagnosis System

\section{Database}

The data model, outlined in Figure 3 was formed along the lines of the DICOM data model of the real world. The figure illustrates that there may be two types of series - unprocessed (original) and processed (secondary captures). 


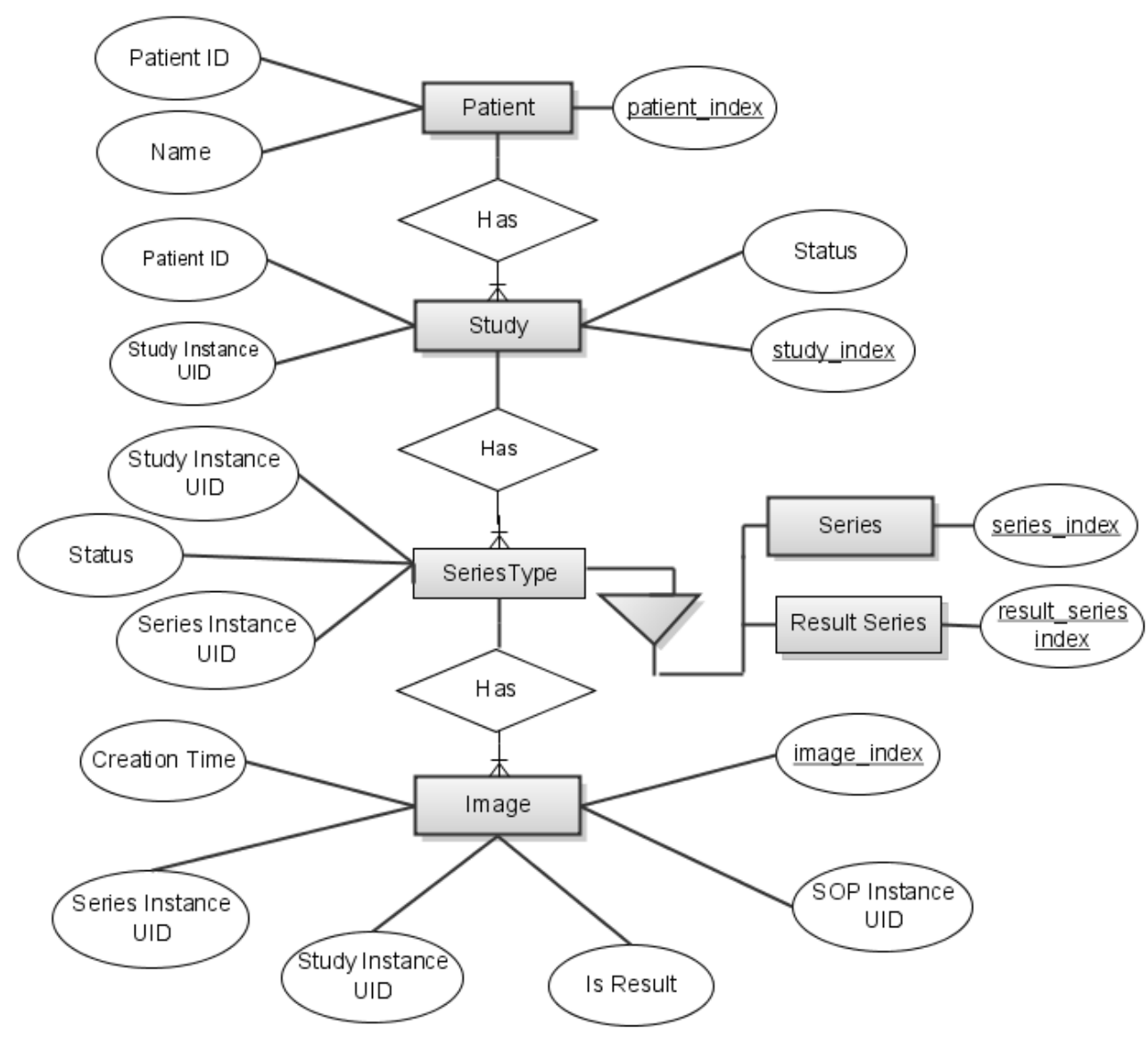

Figure 3: The data model for the CAD application developed along the lines of the DICOM model of the real world, with a few additional non DICOM parameters. As shown, there are two types at the series level - the original, unprocessed series and the resulting, processed series.

\section{File System}

The storage system is divided into two main partitions - the raw repository, which gathers all the DICOM files received from the CT/ PACS; and the "sorted" repository, which contains the same files as in the raw repository organized in a file-folder hierarchy which mimics the DICOM model of the real world.

\section{Control Service}

The Control Service is the heart of the automated CAD system, and recurs at a time interval specified in the configuration file, coordinating dataflow between individual components of the system. It sorts the incoming files into an appropriate hierarchy of folders; catalogues DICOM metadata and other non-DICOM attributes in the database; initiates the running of the algorithm at regular time intervals by keeping track of which studies are complete and ready for processing; thus regulating the automation.

\section{6. (i) GUI}

The graphical user interface provides a view of the original unprocessed series juxtaposed with the secondary capture series, and the mouse wheel is programmed to navigate through the images in a series. The interface can be used both for 
viewing the results generated by the control service, or to intercede and run a series through the CAD manually. The study list is programmed to update itself automatically every 5 minutes, and can also be refreshed manually.

\section{Web Server}

The web server will enable viewing of CAD results remotely, eliminating the need for users to physically man the workstation. This piece of the application is currently in a developmental stage.

\subsection{Workflow}

The green arrows and alphabetical indicators in Figure 2 illustrate the workflow of an automated CAD system.

A. Head CT studies are sent by the PACS to the DICOM Receiver unit in the workstation. Alternatively, studies may be imported from a CD ROM manually via the GUI into the temporary storage area.

B. The DICOM Receiver passes the studies to the Pre-processing Unit which removes and flags suspicious files. If studies are imported from a CD ROM drive, the user needs to carry out this step manually.

C. Files cleared by the Pre Processing unit are then routed to a temporary storage sector on the file system, where they lie unsorted.

D. Studies situated in the temporary storage area are then pulled up by the control service, parsed, sorted, and placed in the permanent section of the file system.

E. Metadata retrieved in step D is inserted into the database.

F. The control service directs the algorithm in the AIH module to pull up specific studies from the storage area, process them, and save the results at a particular location.

G. The algorithm processes the required studies as directed by the Control Service in step F, and saves the results.

H. The user runs the GUI.

I. The GUI queries the database for existing studies and creates a work-list.

J. The GUI retrieves studies and results from the file system as per the user's requests.

K. Users access the system through a web GUI.

L. The web server routes queries to the database and returns the results.

M. The web server retrieves appropriate files from the file system for remote users to view.

\subsection{Technical Specifications}

The system runs on a Windows XP/Vista platform, and requires the .NET Framework Run Time Environment. The workstation will also require a MySQL server. The DICOM receiver module from DCM4CHE was utilized. The control service was built in Visual C\#, and incorporates the original MATLAB CAD code compiled to a dynamic link library.

\section{MULTI-SITE VALIDATION AND EVALUATION}

To verify the efficacy of the CAD algorithm, a two part validation study was conducted. The first part of the study dealt with cases from the Los Angeles County/University of Southern California Hospital System, while the second part dealt with cases from Walter Reed Army Medical Center, Washington DC. These cases were matched with an equal number of normal control cases and processed by the CAD program. The results were then analyzed in order to identify trouble 
spots which need fine-tuning, to provide insight into developing a CAD package for installation at a clinical site, and most importantly, to ascertain the effectiveness of the algorithm.

Performance of the algorithm was evaluated both on a per patient and on a per lesion basis. Evaluation on a per patient basis is of greater clinical relevance because the presence or absence of AIH dictates triage and further clinical management. On a per patient basis, if at least one lesion was correctly identified, the case was counted as one amongst the true positives irrespective of whether the rest of the lesions were false positives or not. If the algorithm detected AIH, but there was none, the case was classified as a false positive. If the algorithm did not detect lesions which did exist, the case was counted as a false negative, and if it did not detect lesions where none were present, that counted as a true negative. The gold standard for determining presence of lesions was the radiologists' report.

Another factor that demands consideration is that the algorithm correctly identified lesions at times, but incorrectly classified them as not being AIH. This could arise due to incorrect registration, which would then lead to incorrect application of the knowledge based rules, ultimately resulting in false negatives.

\subsection{Evaluation at LAC/ USC}

One hundred and thirty five CT head studies of patients diagnosed with AIH were obtained from LAC/USC and matched with a hundred and thirty five normal cases (also from LAC/USC). These studies were captured with a Picker PQ 5000 or 6000 single-slice CT scanner with $5 \mathrm{~mm}$ collimation, at $130 \mathrm{kV}$, and with beam currents of $30 \mathrm{~mA}$.

\section{Results}

Table 2: Summary of CAD results for LAC/USC cases on per patient and per lesion bases [4]

\begin{tabular}{|l|l|l|}
\hline & Overall Sensitivity & $\begin{array}{l}\text { Sensitivity after considering correctly identified by } \\
\text { incorrectly categorized lesions }\end{array}$ \\
\hline Per patient basis & $77 \%$ & $89.6 \%$ \\
\hline Per lesion basis & $69.6 \%$ & $84.2 \%$ \\
\hline
\end{tabular}

The control cases yielded a $100 \%$ false positive result majorly around the falx, most likely due to incorrect registration and alignment.

\subsection{Evaluation at WRAMC}

Eight CT head studies of patients diagnosed with AIH were obtained from WRAMC and matched with eight normal cases (also from WRAMC). Another 13 CT head studies of patients diagnosed with AIH and matched with 13 normal cases has been obtained and are currently being evaluated at the time of the writing of this report.

\section{Results}

Table 3: Summary of CAD results for WRAMC cases on a per patient basis

\begin{tabular}{|l|l|}
\hline True Positives & $10 / 10$ \\
\hline False Positives & $7 / 10$ \\
\hline True Negatives & $1 / 10$ \\
\hline False Negatives & $0 / 10$ \\
\hline
\end{tabular}


Table 4: Summary of CAD results for WRAMC cases on per patient and per lesion bases

\begin{tabular}{|l|l|l|}
\hline & Overall Sensitivity & $\begin{array}{l}\text { Sensitivity after considering correctly } \\
\text { identified by incorrectly categorized lesions }\end{array}$ \\
\hline $\begin{array}{l}\text { Per patient } \\
\text { basis }\end{array}$ & $100 \%$ & $100 \%$ \\
\hline $\begin{array}{l}\text { Per lesion } \\
\text { basis }\end{array}$ & $100 \%$ & $100 \%$ \\
\hline
\end{tabular}

The control cases yielded a $87.5 \%$ false positive result majorly around the falx, likely due to reasons mentioned above.

\subsection{Conclusion}

The system was tested with cases from both LA County Hospital and Walter Reed Army Medical Center. The results generated by the automated system match the results generated by the fundamental MATLAB encoded program. Consequently, a conclusion may be drawn that the new system retains the integrity of the original system while significantly improving the clinical and research workflow efficiency.

Although the initial sample size obtained from WRAMC is small, the CAD system was highly sensitive in detecting AIH. At this time it is difficult to make a comparison between those studies obtained from LAC/USC and WRAMC due to the small size of the WRAMC image data set. Such comparison can be done in the future as the WRAMC dataset is increasing and currently being evaluated by the CAD system.

\section{FUTURE WORK}

In summary, a Computer Aided Diagnosis algorithm has been developed and validated across two clinical sites, and incorporated into a system which automates the workflow, facilitating its utilization in a clinical environment. Future

work includes installing the system at WRAMC to carry out a small pilot study for evaluating both the algorithm and the system.

\section{REFERENCES}

[1] Perry JJ, et al., "Attitudes and judgment of emergency physicians in the management of patients with acute headache," Acad Emerg Med 12(1), 33-7 (2005).

[2] Perry JJ, et al., "Attitudes and judgment of emergency physicians in the management of patients with acute headache," Acad Emerg Med 12(1), 33-7 (2005).

[3] Mader TJ, Mandel A., "A new clinical scoring system fails to differentiate hemorrhagic from ischemic stroke when used in the acute care setting," J Emerg Med 16(1), 9-13 (1998).

[4] Chan T., "Computer aided detection of small acute intracranial hemorrhage on computer tomography of brain," Computerized medical Imaging and Graphics 31, 285-298 (2007).

[5] Joon K. Lee, et al., "Evaluation of a computer-aided detection algorithm for timely diagnosis of small acute intracranial hemorrhage on computed tomography in a critical care environment." 